\title{
BAU 2013: Nachhaltigkeit bestimmt das Handeln
}

Wenn die Bau 2013, Weltleitmesse für Architektur, Materialien, Systeme, vom 14. bis 19. Januar 2013 auf dem Gelände der Neuen Messe München ihre Tore öffnet, werden auch Kleb- und Dichtstoffunternehmen mit von der Partie sein.

Z

u den Leitthemen dieser Messe zählt die Nachhaltigkeit.

Beim Thema Nachhaltigkeit hat sich in den vergangenen Jahren viel getan. Dass der Begriff den gesamten Lebenszyklus von Gebäuden umfasst (von der Planung über die Nutzung bis zum Abriss) und neben ökologischen auch ökonomische und soziokulturelle Aspekte beinhaltet, darüber herrscht weitgehend Einigkeit. In aller Welt wurden entsprechende Bewertungssysteme zur Zertifizierung nachhaltiger Gebäude entwickelt.

Aber nicht nur komplette Gebäude, sondern auch die Bauteile und Materialien, aus denen sie bestehen, werden auf ihre Nachhaltigkeit hin geprüft. Umweltproduktdeklarationen (EPDs) sollen künftig Informationen über die funktionale und ökologische Qualität von Bauteilen und Baustoffen liefern.

Viele Aussteller - so auch die Aussteller auf dem Gebiet der Bauchemie - werden ihre Präsentationen auf dieses Thema ausrichten und entsprechende Lösungen vorstellen.

Die Bauchemie inklusive der Kleb- und Dichtstoffe hat auf der Bau eine lange Tradition, da sie die Entwicklung und Verwendung heutiger Materialien erst ermöglicht. „Über die verschiedenen Produktbereiche hinweg werden Themen wie Nachhaltigkeit und Ressourcenschutz die aktuellen Ausstellungstrends der Bau 2013 prägen“, prognostiziert Norbert Schröter, Hauptgeschäftsführer Deutsche Bauchemie e.V. „Das langsame Wiedererstarken des Neubaus erweitert neben dem traditionell starken Sanierungsbereich die Möglichkeiten der bauchemischen Industrie, in München ihre Innovationen einem größeren Interessentenkreis vorzustellen. “ Dazu gehören beispielsweise widerstandsfähige Reparaturmörtel, die auch bei Tieftemperaturen die dauerhafte Instandsetzung von Betonflächen ermöglichen, oder schnell abbindende SicherheitsDichtschlämme, die für die rissüberbrückende Abdichtung von Keramikbelägen in Duschen und Schwimmbecken oder bei Fundamenten sorgen. Neben Kleb- und Dichtstoffen sowie Oberflächenbehandlungen wie Putze, Lacke und Farben werden auch zahlreiche Isolier- und Dämmstoffe für den Wärme-, Kälte-, Brand- und Schallschutz präsentiert.

\section{Hersteller von Kleb- und Dichtstoffen auf der Bau 2013}

Bostik GmbH

BoTec $\mathrm{GmbH}$

A4.428

Enke-Werk Joh. Enke GmbH \& Co. KG

A5.239

ferax e.K.

B6.341

fischer Deutschland Vertriebs GmbH A1.329

Forbo Erfurt GmbH

A5.341

Fraunhofer-Institut für Fertigungstechnik und Angewandte Materialforschung IFAM C2.131 Gerlinger GmbH \& Co. KG A5.119

ISO-Chemie GmbH A5.432

Kiesel Bauchemie GmbH u. Co. KG A5.139

Kömmerling Chemische Fabrik GmbH A3.523

MEM Bauchemie GmbH A5.426

Moll bauökologische Produkte GmbH A5.200

Otto-Chemie Hermann Otto GmbH A5.410

Ramsauer $\mathrm{GmbH} \&$ Co. KG A5.416

Schönox $\mathrm{GmbH} \quad$ A6.112 und B6.523

Schomburg A5.221

Sika Deutschland GmbH A1.530

Tremco illbruck GmbH \& Co. KG A2.111

Uzin Utz AG

$A 4.402$ und $A 4.502$

Wagener Bauartikel GmbH A4.430

Webac-Chemie GmbH A5.231

Weiss Chemie + Technik GmbH \& Co. KG A5.219

\section{Termin und Ort der WCARP 2014 stehen fest}

Termin und Ort der nächsten Weltklebstoffkonferenz stehen fest. Die WCARP - 5th World Congress on Adhesion and Related Phenomena wird vom 7. bis 10.9.2014 in Nara, Japan, stattfinden.

$D^{\text {ses }}$ ieses viertägige internationale Event wird im vierjährigen Rhythmus veranstaltet und bringt führende internationale Experten auf dem Gebiet der Adhäsionsforschung zusammen, um sich über den neusten Stand auf diesem Gebiet auszutauschen.
Das Organisationskomitee setzt sich aus folgenden Gesellschaften zusammen: the Adhesion Society (USA), the Adhesion Society in Spain (Spain), Beijing Adhesion Society (China), DECHEMA e.V. (Germany), Société Française du Vide (France), the Society of Adhe- sion and Adhesives (United Kingdom), the Society of Adhesion and Interfaces (Korea).

Weitere Infos: www.adhesion.or.jp/ wcarp 